American J. of Engineering and Applied Sciences 3 (1): 56-63, 2010

ISSN 1941-7020

(C) 2010 Science Publications

\title{
Anisotropy Influence of Cubic Solid on Dynamic Hertzian Contact Stiffness for a Vibrating Rigid Indenter
}

\author{
Jiayong Tian \\ Institute of Crustal Dynamics, China Earthquake Administration, \\ P.O. Box 2855, Beijing, Republic of China, 100085
}

\begin{abstract}
Problem statement: Resonance-type microscopies have been widely used to evaluate the nanoscaled or microscaled surface elastic properties of materials by the resonance-frequency shifts of an oscillator, which contacts the surface of materials by a spherical tip. Approach: The tip-specimen contact is modeled to be a spring support, whose stiffness is given by the traditional Hertzian contact theory. However, because of the influence of the oscillator vibration and the anisotropy in nanoscaled or microscaled region of materials, the predicted results from the traditional Hertzian contact theory can not coincide with the experimental observations. In order to explain this discrepancy, dynamic contact stiffness at the contact interface between a rigid sphere and a semi-infinite cubic solid is investigated. Results: An oscillating force being superposed on a biasing force excites the oscillation of the sphere contacting with the solid surface, which causes the contact radius to vary with the oscillation. The assumption of sufficiently small oscillating force compared with the biasing force yields an oscillating-contact-pressure distribution of the constant contact radius and then dynamic contact stiffness. Because the oscillating-contact-pressure distribution cannot promise the uniform contact deformation, the influence of contact-displacement conditions is discussed. Conclusion: It is shown that dynamic contact stiffness depends on the oscillating frequency and contact radius of the sphere and the solid anisotropy.
\end{abstract}

Key words: Anisotropy, dynamic contact, cubic solid, rigid indenter, contact deformation

\section{INTRODUCTION}

The quantitative evaluation of the surface elastic properties of materials in nanoscaled or microscaled is of great importance in material engineering (Kester et al., 1999), which can be determined by Atomic Force Microscopy (AFM) (Yamanaka and Nakano, 1998; Yamanaka et al., 1999; Rabe et al., 1998) and Resonance Ultrasound Microscopy (RUS) (Ogi et al., 2003; Tian et al., 2004; Tian et al., 2008). In these microscopies, an applied biasing force make an oscillator touch the surface of materials through a tip and a piezoelectric transducer or dynamic magnetic filed excites the vibration of the oscillator. The local surface elastic properties and degradation of materials are reflected from resonance-frequency shifts of the oscillator. The tip-specimen contact was usually modeled to be a spring support, whose stiffness is given by the traditional Hertzian contact theory (Johnson, 1985). The traditional Hertzian contact theory provides the static contact stiffness for the contacting isotropic materials. But all materials exhibit the anisotropy in nanoscaled or microscaled range (Simmons and Wang, 1971). Furthermore, the vibration of the oscillator has great influence on the contact stiffness at the contact interface because the contact effect is sensitive to the contact deformation at the contact interface. These evidences show that the static contact model may fails in deducing the elastic constants of materials from the resonance-frequency shifts of the oscillator in resonance-type microscopies, which has been verified by the experiments (Tian et al., 2004). Therefore, the evaluation of dynamic Hertzian contact for anisotropic solids is a key issue in resonance-type microscopies.

For the design of dynamic machine foundation in civil engineering, the vibration of a rigid punch against a semi-infinite solid has been investigated extensively by considering the dynamic contact deformation (Sung, 1953; Barkan, 1962; Bycrot, 1956; Awojobi and Grootenhuis, 1965; Luco and Westmann, 1971; Veletsos and Verbic, 1974), where the contact radius keeps constant and the uniform dynamic contact deformation at the contact interface equals the indentation of the punch (contact-displacement condition). Two methods have been adopted to solve this problem. The one is that the prescribed contactpressure distribution gives the dynamic contact deformation by the consideration of wave propagation in the solid (Sung, 1953; Barkan, 1962; Bycrot, 1956). However, the contact displacement condition is 
satisfied only at a certain point of the contact interface. The other is that the contact-displacement condition with the unknown dynamic contact pressure distribution yields a dual integral equation (Awojobi and Grootenhuis, 1965; Luco and Westmann, 1971; Veletsos and Verbic, 1974). As for Hertzian contact vibration, the contact radius varies as the oscillation of the sphere, which can be attributed to be a nonlinear contact problem. Tian et al. (2004) studied dynamic Hertzian contact stiffness at the contact interface between a spherical tip and an isotropic solid. Assuming that the oscillating force is much smaller than the biasing force, they simplified this problem into the superimposition of a static problem and a dynamic contact problem of the constant contact radius, which induces the prescribed dynamic contact pressure distribution with the unknown disturbance variables of the contact radius and the maximum of the contact pressure. The contact displacement conditions are satisfied at the center and the edge of the contact interface, which is named as Two Points (TP) model. However, the TP model yields the negative contact stiffness for high-frequency oscillation, which is unreasonable.

In this study, we introduce a dynamic-contactpressure distribution with the unknown disturbance term of the contact radius to investigate dynamic contact stiffness of a rigid sphere against a semiinfinite cubic solid, considering the influence of the contact-displacement conditions. First, if the oscillating force is much smaller than the applied biasing force, the nonlinear dynamic contact problem is simplified into the superimposition of a static contact problem and a constant-contact-radius dynamic contact problem, which induces the oscillating-contact-pressure distribution with the unknown disturbance variable of the contact radius. Second, we deduce dynamic contact stiffness by considering dynamic contact deformation and dynamic contact displacement condition. Third, we discuss the influence of contact-displacement conditions on the dynamic Hertzian contact stiffness. Lastly, we further discuss the influence of the vibrating frequency and contact radius of the rigid sphere and elastic properties of the cubic solids on the dynamic contact stiffness.

\section{MATERIALS AND METHODS}

The vibration of a rigid sphere against a semi-infinite cubic solid: A biasing force $F$ presses a rigid sphere of radius $\mathrm{R}$ to contact frictionlessly with a semi-infinite cubic solid of mass density $\rho$, which is shown in Fig. 1. A harmonic force $\delta \mathrm{Fe}^{\mathrm{i} \omega \mathrm{t}}$ being superimposed upon the biasing force excites the sphere oscillation normally on the solid surface, where $\omega$ is angular frequency.

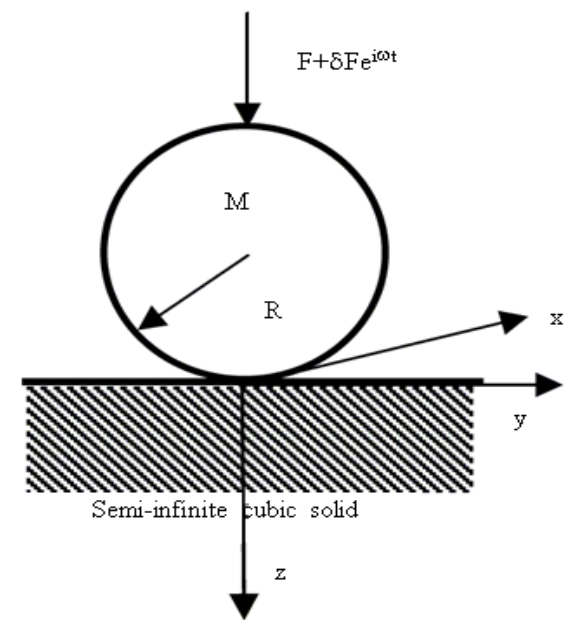

Fig. 1: The vibration of a rigid sphere against on a semi-infinite cubic solid acted by a biasing force and an oscillating force

A Cartesian coordinates $(\mathrm{x}, \mathrm{y}, \mathrm{z})$ whose origin is located at contact point is set to make $x-y$ plane coincide with contact tangent plane and the $\mathrm{z}$ axis along the inward normal of the solid.

Oscillating-contact-pressure distribution: First, we consider the static contact of the sphere and the solid subjected to the biasing force $\mathrm{F}$, which yields a circular contact interface of radius a as (Tian et al., 2005):

$\mathrm{a}=\sqrt[3]{\frac{3 \mathrm{FRI}_{1}}{8 \pi}}$

The contact pressure distribution $\mathrm{p}(\mathrm{x}, \mathrm{y})$ and the indentation $\mathrm{w}$ are denoted as, respectively (Tian et al., 2005):

$\mathrm{p}(\mathrm{x}, \mathrm{y})=\frac{4 \mathrm{a}}{\mathrm{RI}_{1}} \sqrt{1-\left(\mathrm{x}^{2}+\mathrm{y}^{2}\right) / \mathrm{a}^{2}}$

$\mathrm{w}=\frac{\mathrm{I}_{0}}{8} \sqrt[3]{9 \mathrm{~F}^{2} / \pi^{2} \mathrm{RI}}$

Where:

$I_{0}=\int_{0}^{2 \pi} \hat{G}(\cos \theta, \sin \theta) d \theta$

$I_{1}=\int_{0}^{2 \pi} \hat{G}(\cos \theta, \sin \theta) \cos ^{2} \theta d \theta$

Here, $\hat{\mathrm{G}}\left(\xi_{1}, \xi_{2}\right)$ is Fourier transform of Green function $\mathrm{G}\left(\mathrm{x}_{1}, \mathrm{x}_{2}\right)$. The static-contact stiffness $\mathrm{K}_{\mathrm{sa}}$ is derived from Eq. 3: 
$\mathrm{K}_{\mathrm{sa}}=\mathrm{dF} / \mathrm{dw}=4 \sqrt[3]{3 \mathrm{FR} \pi^{2} \mathrm{I}_{1} / \mathrm{I}_{0}^{3}}$

Next, the harmonic force $\delta \mathrm{Fe}^{\mathrm{i} \omega \mathrm{t}}$ is superimposed to the static force to excite the sphere oscillation, where the contact radius varies with the oscillation. Introducing the perturbation term $\delta a(t)$ of the contact radius, dynamic contact pressure distribution is written similarly as:

$\mathrm{p}(\mathrm{x}, \mathrm{y}, \mathrm{t})=\frac{4(\mathrm{a}+\delta \mathrm{a}(\mathrm{t}))}{\mathrm{RI}_{1}} \sqrt{1-\left(\mathrm{x}^{2}+\mathrm{y}^{2}\right) /(\mathrm{a}+\delta \mathrm{a}(\mathrm{t}))^{2}}$

where, $\delta \mathrm{a}(\mathrm{t}) \approx \delta \mathrm{a}^{\mathrm{i} \omega t}$ and $\delta \mathrm{a}$ is unknown constant satisfying $|\delta \mathrm{a}|<<\mathrm{a}$. Neglecting higher-order terms, Eq. 5 can be simplified into:

$\mathrm{p}(\mathrm{x}, \mathrm{y}, \mathrm{t}) \approx \mathrm{p}_{0} \sqrt{1-\left(\mathrm{x}^{2}+\mathrm{y}^{2}\right) / \mathrm{a}^{2}}+\delta \mathrm{f}(\mathrm{x}, \mathrm{y}) \mathrm{e}^{\mathrm{i} \omega t}$

where $\delta f(x, y) e^{i \omega t}$ represents the oscillating contactpressure distribution given by:

$$
\delta f(x, y)=\frac{4 \delta a}{R_{1}} \frac{1}{\sqrt{1-\left(x^{2}+y^{2}\right) / a^{2}}}
$$

It is shown from Eq. 6 that the dynamic contactpressure distribution of nonconstant contact radius in Eq. 5 can be expressed to be the superimposition of a static contact pressure distribution and a harmonic dynamic contact pressure distribution of the constant contact radius. Therefore, only the harmonic dynamic response will be considered in the following analysis.

Introducing the dynamic indentation of the sphere:

$w(t)=w+\delta w e^{i \omega t}$

the equation of motion can be denoted as:

$$
M \ddot{w}(t)=F+\delta F^{i \omega t}-\int_{-\infty}^{\infty} \int_{-\infty}^{\infty} p(x, y, t) d x d y
$$

where, M is the sphere mass. Substitution of Eq. 6 and 7 into Eq. 8 yields:

$$
-\omega^{2} \mathrm{M} \delta \mathrm{w}=\delta \mathrm{F}-\delta \mathrm{P}
$$

where, $\delta \mathrm{P}=\frac{8 \pi \mathrm{a}^{2} \delta \mathrm{a}}{\mathrm{RI}_{1}}$ is the oscillating elastic contact force.
Response of the semi-infinite cubic solid subjected to the oscillating contact pressure distribution: Next, we consider surface displacements in the semiinfinite cubic solid caused by the oscillating pressure distribution $\delta \mathrm{f}(\mathrm{x}, \mathrm{y}) \mathrm{e}^{\mathrm{i} \omega \mathrm{t}}$, where the term $\mathrm{e}^{\mathrm{i} \omega \mathrm{t}}$ will be neglected in the following analysis. Displacement components $\mathrm{u}_{\mathrm{x}}(\mathrm{x}, \mathrm{y}, \mathrm{z}), \mathrm{u}_{\mathrm{y}}(\mathrm{x}, \mathrm{y}, \mathrm{z})$ and $\mathrm{u}_{\mathrm{z}}(\mathrm{x}, \mathrm{y}, \mathrm{z})$ satisfy the following equations of motion (Nayfeh, 1995):

$$
\left.\begin{array}{l}
C_{11} u_{x, x x}+C_{66} u_{x, y y}+C_{66} u_{x, z z} \\
+\left(C_{12}+C_{66}\right) u_{y, x y}+\left(C_{12}+C_{66}\right) u_{z, x z}+\rho \omega^{2} u_{x}=0 \\
C_{11} u_{y, y y}+C_{66} u_{y, x x}+C_{66} u_{y, z z} \\
+\left(C_{12}+C_{66}\right) u_{x, x y}+\left(C_{12}+C_{66}\right) u_{z, y z}+\rho \omega^{2} u_{y}=0 \\
C_{11} u_{z, z z}+C_{66} u_{z, x x}+C_{66} u_{z, y y} \\
+\left(C_{12}+C_{66}\right) u_{x, x z}+\left(C_{12}+C_{66}\right) u_{y, y z}+\rho \omega^{2} u_{z}=0
\end{array}\right\}
$$

and

$$
\left[\begin{array}{c}
\tau_{\mathrm{xx}} \\
\tau_{\mathrm{yy}} \\
\tau_{\mathrm{zz}} \\
\tau_{\mathrm{yz}} \\
\tau_{\mathrm{zx}} \\
\tau_{\mathrm{xy}}
\end{array}\right]=\left[\begin{array}{cccccc}
\mathrm{C}_{11} & \mathrm{C}_{12} & \mathrm{C}_{12} & 0 & 0 & 0 \\
\mathrm{C}_{12} & \mathrm{C}_{11} & \mathrm{C}_{12} & 0 & 0 & 0 \\
\mathrm{C}_{12} & \mathrm{C}_{12} & \mathrm{C}_{11} & 0 & 0 & 0 \\
0 & 0 & 0 & \mathrm{C}_{66} & 0 & 0 \\
0 & 0 & 0 & 0 & \mathrm{C}_{66} & 0 \\
0 & 0 & 0 & 0 & 0 & \mathrm{C}_{66}
\end{array}\right]
$$

$$
\left[\begin{array}{ccc}
\partial / \partial x & 0 & 0 \\
0 & \partial / \partial y & 0 \\
0 & 0 & \partial / \partial z \\
0 & \partial / \partial z & \partial / \partial y \\
\partial / \partial z & 0 & \partial / \partial x \\
\partial / \partial y & \partial / \partial x & 0
\end{array}\right]\left[\begin{array}{l}
u_{x} \\
u_{y} \\
u_{z}
\end{array}\right]
$$

The boundary conditions at $\mathrm{z}=0$ are expressed as:

$$
\left.\begin{array}{rl}
\tau_{z z} & =-\delta f(x, y), \text { for } \sqrt{x^{2}+y^{2}} \leq a \\
\tau_{z x} & =0 \\
\tau_{z y} & =0
\end{array}\right\}
$$

We apply the double Fourier transform to this problem, which is defined as:

$F\left(\xi_{1}, \xi_{2}, z\right)=\int_{-\infty}^{\infty} \int_{-\infty}^{\infty} f(x, y, z) e^{-i \xi_{1} x-i \xi_{2} y} d x d y$

Application of the double Fourier transform to Eq. 10-12 yields: 


$$
\begin{aligned}
& \left(\mathrm{C}_{11} \xi_{1}^{2}+\mathrm{C}_{66} \xi_{2}^{2}\right) \hat{\mathrm{u}}_{\mathrm{x}}-\mathrm{C}_{66} \hat{\mathrm{u}}_{\mathrm{x}, \mathrm{zz}} \\
& +\xi_{1} \xi_{2}\left(C_{12}+C_{66}\right) \hat{u}_{y}-i \xi_{1}\left(C_{12}+C_{66}\right) \hat{u}_{z, z}-\rho \omega^{2} \hat{u}_{x}=0 \\
& \xi_{1} \xi_{2}\left(C_{12}+C_{66}\right) \hat{u}_{x}+\left(C_{66} \xi_{1}^{2}+C_{11} \xi_{2}^{2}\right) \hat{u}_{y}- \\
& \mathrm{C}_{66} \hat{\mathrm{u}}_{\mathrm{y}, z \mathrm{z}}-\mathrm{i} \xi_{2}\left(\mathrm{C}_{12}+\mathrm{C}_{66}\right) \hat{\mathrm{u}}_{z, \mathrm{z}}-\rho \omega^{2} \hat{\mathrm{u}}_{\mathrm{y}}=0 \\
& -i \xi_{1}\left(C_{12}+C_{66}\right) \hat{u}_{x, z}-i \xi_{2}\left(C_{12}+C_{66}\right) \hat{u}_{y, z} \\
& +\mathrm{C}_{66}\left(\xi_{1}^{2}+\xi_{2}^{2}\right) \hat{\mathrm{u}}_{\mathrm{z}}-\mathrm{C}_{11} \hat{\mathrm{u}}_{\mathrm{z}, \mathrm{zz}}-\rho \omega^{2} \hat{\mathrm{u}}_{\mathrm{z}}=0 \\
& {\left[\begin{array}{l}
\hat{\tau}_{\mathrm{xx}} \\
\hat{\tau}_{\mathrm{yy}} \\
\hat{\tau}_{\mathrm{zz}} \\
\hat{\tau}_{\mathrm{yz}} \\
\hat{\tau}_{\mathrm{zx}} \\
\hat{\tau}_{\mathrm{xy}}
\end{array}\right]=\left[\begin{array}{cccccc}
\mathrm{C}_{11} & \mathrm{C}_{12} & \mathrm{C}_{12} & 0 & 0 & 0 \\
\mathrm{C}_{12} & \mathrm{C}_{11} & \mathrm{C}_{12} & 0 & 0 & 0 \\
\mathrm{C}_{12} & \mathrm{C}_{12} & \mathrm{C}_{11} & 0 & 0 & 0 \\
0 & 0 & 0 & \mathrm{C}_{66} & 0 & 0 \\
0 & 0 & 0 & 0 & \mathrm{C}_{66} & 0 \\
0 & 0 & 0 & 0 & 0 & \mathrm{C}_{66}
\end{array}\right]} \\
& {\left[\begin{array}{ccc}
\mathrm{i} \xi_{1} & 0 & 0 \\
0 & \mathrm{i} \xi_{2} & 0 \\
0 & 0 & \partial / \partial \mathrm{z} \\
0 & \partial / \partial \mathrm{z} & \mathrm{i} \xi_{2} \\
\partial / \partial \mathrm{z} & 0 & \mathrm{i} \xi_{1} \\
\mathrm{i} \xi_{2} & \mathrm{i} \xi_{1} & 0
\end{array}\right]\left[\begin{array}{l}
\hat{\mathrm{u}}_{\mathrm{x}} \\
\hat{\mathrm{u}}_{\mathrm{y}} \\
\hat{\mathrm{u}}_{\mathrm{z}}
\end{array}\right]}
\end{aligned}
$$

and

$$
\left.\begin{array}{l}
\hat{\tau}_{z z}=-\frac{8 \pi \mathrm{a}^{2} \sin (\eta)}{\mathrm{RI}_{1} \eta} \\
\hat{\tau}_{\mathrm{zx}}=0 \\
\hat{\tau}_{\mathrm{zy}}=0
\end{array}\right\} \text {, at } \mathrm{z}=0
$$

where, $\eta=\mathrm{a} \sqrt{\xi_{1}^{2}+\xi_{2}^{2}}$.

The general solutions for Eq. 14 satisfying far-field condition can be written as:

$$
\hat{\mathrm{W}}_{\mathrm{k}}\left(\xi_{1}, \xi_{2}, \mathrm{z}\right)=\sum_{\alpha=1}^{3} \mathrm{~A}_{\mathrm{k}}^{\alpha} \mathrm{e}^{\mathrm{im}_{\alpha} \mathrm{z}} \mathrm{b}_{\alpha}, \mathrm{k}=1,2,3
$$

where, $\mathrm{m}_{\alpha}$ has positive imaginary part. $\mathrm{m}_{\alpha}$ and $\mathrm{A}_{\mathrm{k}}^{\alpha}$ are determined by:

$$
\begin{aligned}
& {\left[\begin{array}{cc}
\mathrm{C}_{11} \xi_{1}^{2}+\mathrm{C}_{66} \xi_{2}^{2}+\mathrm{C}_{66} \mathrm{~m}_{\alpha}^{2}-\rho \omega^{2} \\
\xi_{1} \xi_{2}\left(\mathrm{C}_{12}+\mathrm{C}_{66}\right) & \xi_{1} \mathrm{~m}_{\alpha}\left(\mathrm{C}_{12}+\mathrm{C}_{66}\right) \\
\mathrm{C}_{66} \mathrm{~m}_{\alpha}^{2}+\mathrm{C}_{66} \xi_{1}^{2}+\mathrm{C}_{11} \xi_{2}^{2}-\rho \omega^{2} & \xi_{2} \mathrm{~m}_{\alpha}\left(\mathrm{C}_{12}+\mathrm{C}_{66}\right) \\
\mathrm{C}_{66} \xi_{1}^{2}+\mathrm{C}_{66} \xi_{2}^{2}+\mathrm{C}_{11} \mathrm{~m}_{\alpha}^{2}-\rho \omega^{2}
\end{array}\right]} \\
& \left\{\begin{array}{l}
\mathrm{A}_{1}^{\alpha} \\
\mathrm{A}_{2}^{\alpha} \\
\mathrm{A}_{3}^{\alpha}
\end{array}\right\}=\left\{\begin{array}{l}
0 \\
0 \\
0
\end{array}\right\}
\end{aligned}
$$

Coefficients $b_{\alpha}$ are given by point-force boundary conditions as:

$$
\left.\begin{array}{l}
\sum_{\alpha=1}^{3}\left(A_{1}^{\alpha} m_{\alpha}+A_{3}^{\alpha} \xi_{1}\right) b_{\alpha}=0 \\
\sum_{\alpha=1}^{3}\left(A_{2}^{\alpha} m_{\alpha}+A_{3}^{\alpha} \xi_{2}\right) b_{\alpha}=0 \\
\sum_{\alpha=1}^{3}\left(\frac{C_{12}}{C_{11}} A_{1}^{\alpha} \xi_{1}+\frac{C_{12}}{C_{11}} A_{2}^{\alpha} \xi_{2}+A_{3}^{\alpha} m_{\alpha}\right) b_{\alpha}=-i
\end{array}\right\}
$$

After solving $b_{\alpha}$ from Eq. 19, the surface normal displacement $\hat{\mathrm{u}}_{\mathrm{z}}(\eta, \phi)$ is expressed as:

$\hat{\mathrm{u}}_{\mathrm{z}}(\eta, \phi)=-\frac{8 \pi \mathrm{a}^{3}}{\mathrm{C}_{11} \mathrm{RI}_{1}} \hat{\mathrm{W}}_{3}(\eta \cos (\phi), \eta \sin (\phi)) \frac{\sin (\eta)}{\eta}$

Application of the inverse double Fourier transform to Eq. 20 yields:

$\mathrm{u}_{\mathrm{z}}(\tilde{\mathrm{r}}, \theta)=\frac{4 \mathrm{a} \delta \mathrm{a}}{\mathrm{C}_{11} \mathrm{RI}_{1}} \mathrm{~N}(\tilde{\mathrm{r}}, \theta)$

where:

$$
\mathrm{N}(\tilde{\mathrm{r}}, \theta)=-\int_{0}^{\infty} \int_{0}^{2 \pi} \hat{\mathrm{W}}(\eta \cos (\varphi), \eta \sin (\varphi)) \sin (\eta) \mathrm{e}^{\mathrm{in} \tilde{\mathrm{\eta}} \cos (\theta-\varphi)} \mathrm{d} \eta \mathrm{d} \varphi
$$

and

$$
\tilde{r}=\sqrt{\left(x^{2}+y^{2}\right) / a^{2}}
$$

Integrands in the $N(\tilde{r}, \theta)$ have poles along the real axis of $\eta$ plane corresponding to Rayleigh waves. In order to avoid this singularity along the integral path, we introduce complex elastic constants $\hat{\mathrm{C}}_{\mathrm{ij}}=\mathrm{C}_{\mathrm{ij}}\left(1+\mathrm{i} \mathrm{Q}_{\mathrm{ij}}^{-1}\right)$ to make the poles deviate from the real axis of $\eta$ plane (Cornejo C'ordova, 2002). $\mathrm{Q}_{\mathrm{ij}}^{-1}$ are internal frictions for elastic constants of the solid. There are usually different independent internal frictions $Q_{i j}^{-1}$ for different elastic constants in the solid. Here we assume $\mathrm{Q}_{\mathrm{ij}}^{-1}$ to be the same value $Q^{-1}=0.02$ to simplify the analysis.

Dynamic displacement conditions at the contact interface: In order to obtain $\delta a, \delta w$ and then $u_{z}(x, y)$, additional condition is required. Hertzian contact theory (Johnson, 1985) gives the contact-displacement condition: 
$u_{z}(x, y)+u_{z 0}(x, y)=\delta w+w-\frac{x^{2}+y^{2}}{2 R}$

where, $\mathrm{u}_{\mathrm{z} 0}(\mathrm{x}, \mathrm{y})$ is the static surface normal displacement. Equation 22 provides that the harmonic normal displacement $\mathrm{u}_{\mathrm{z}}(\mathrm{x}, \mathrm{y})$ at the contact interface is uniform and equals the harmonic indentation $\delta \mathrm{w}$ of the sphere:

$\mathrm{u}_{\mathrm{z}}(\tilde{\mathrm{r}}, 0)=\delta \mathrm{w}$

The oscillating-contact-pressure distribution in Eq. 6 can promise the uniform normal displacement of the solid at contact interface for the static contact $(\omega=0)$. However, for the dynamic case $(\omega>0)$, the harmonic normal displacement is impossible to keep uniform at the contact interface. Figure 2 shows that with the increase of the oscillating frequency, the nonuniformity of the harmonic normal displacement distribution becomes increasingly evident. Moreover, Frost, (1974) also investigated a rigid cylinder laid sideways on the edge of a viscoelastic solid and vibrated at about $90 \mathrm{kHz}$ while being pressed with a static force $\mathrm{F}$ onto the test sample and exerting a timeaveraged ultrasonic force $\delta \mathrm{F}$. His experimental observations show that the contact is intermittent for $\delta \mathrm{F} \ll \mathrm{F}$, which has been attributed to the nonuniformity of the dynamic contact deformation. Therefore, the approximated dynamic contact-displacement conditions must be provided to simplify the following analysis.

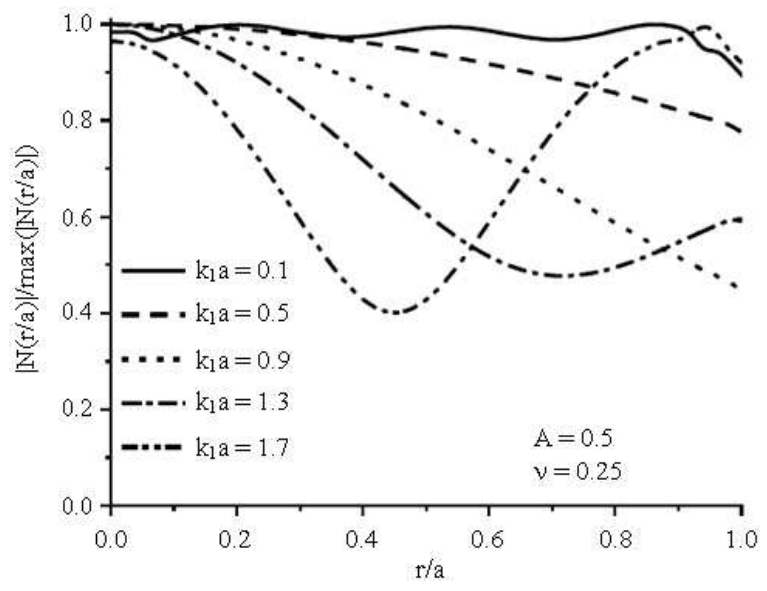

Fig. 2: The oscillating contact deformation distribution at the contact interface caused by the oscillating contact pressure distribution
In the studies of the contact vibration for a rigid punch, Sung, (1953) presented that the contactdisplacement condition in Eq. 23 is satisfied only at the center of the contact interface, which is called the R0 model:

$\mathrm{u}_{\mathrm{z}}(0,0)=\mathrm{w} \delta$

The R1 model means that the normal displacement at the edge of the contact interface equals the indentation:

$\mathrm{u}_{\mathrm{z}}(1,0)=\delta \mathrm{w}$

SA model is that the arithmetic mean of displacements at the center and the edge of the contact interface equal the indentation (Barkan, 1962):

$\left(\mathrm{u}_{\mathrm{z}}(1,0)+\mathrm{u}_{\mathrm{z}}(1,0)\right) / 2=\delta \mathrm{w}$

Bycroft, (1956) gave the WA model, where the weighted average displacement at the contact interface equals the indentation:

$\int_{0}^{1} \frac{\mathrm{u}_{\mathrm{z}}(\mathrm{r}, 0) \mathrm{r}}{\sqrt{1-\mathrm{r}^{2}}} \mathrm{dr}=\delta \mathrm{w}$

Dynamic contact stiffness: Combination of Eq. 9, 21 and 24-27 yields the dynamic contact stiffness $\mathrm{K}_{\mathrm{da}}$, which is defined as the ratio of the oscillating elastic contact force $\delta \mathrm{P}$ to the oscillating indentation $\delta \mathrm{w}$ :

$\mathrm{K}_{\mathrm{da}}= \begin{cases}\frac{2 \pi \mathrm{aC}_{11}}{\mathrm{~N}(0,0)} & \mathrm{R} 0 \text { model } \\ \frac{2 \pi \mathrm{aC}_{11}}{\mathrm{~N}(1,0)} & \mathrm{R} 1 \text { model } \\ \frac{4 \pi \mathrm{aC}_{11}}{\mathrm{~N}(0,0)+\mathrm{N}(1,0)} & \mathrm{RA} \text { model } \\ \frac{2 \pi \mathrm{aC}}{11} & \mathrm{WA} \text { model } \\ \int_{0}^{1} \mathrm{~N}(\mathrm{r}, 0) \mathrm{r} / \sqrt{1-\mathrm{r}^{2}} \mathrm{dr} & \end{cases}$

In order to consider dynamic characteristic of the dynamic anisotropic Hertzian contact, we introduce the Dynamic-Contact-Stiffness Factor (DCSF) $\kappa_{\mathrm{f}}$ related with the static anisotropic contact stiffness $\mathrm{K}_{\mathrm{sa}}$ (Tian et al., 2005) as:

$\kappa_{\mathrm{f}}=\mathrm{K}_{\mathrm{da}} / \mathrm{K}_{\mathrm{sa}}$

The DCSF is influenced by the normalized wave number $\mathrm{k}_{1} \mathrm{a}=\sqrt{\omega^{2} \rho / \mathrm{C}_{11}} \mathrm{a}$ and anisotropic factor $A=2 C_{66} /\left(C_{11}-C_{12}\right)$, Poisson ratio $v=C_{12} /\left(C_{11}+C_{12}\right)$ of the cubic solid. 
Am. J. Engg. \& Applied Sci., 3 (1): 56-63, 2010

\section{RESULTS AND DISCUSSION}

Firstly, Fig. 3 shows the influence of the contactdisplacement-condition models on the DCSFs at $\mathrm{A}=0.5$ and $v=0.25$. The DCSF for the TP model is also shown in Fig. 3. Figure 3a and b denote the real and imaginary parts of DCSF, which are related with the contact stiffness and the radiation damping, respectively. The influence of the contactdisplacement conditions will increase as $\mathrm{k}_{1} \mathrm{a}$ increases. At the higher frequency, the R0, SA, and TP models show the negative contact stiffness and radiation damping, which are unreasonable. The negative contact stiffness and radiation damping means that the vibration of the oscillator will approach instable.

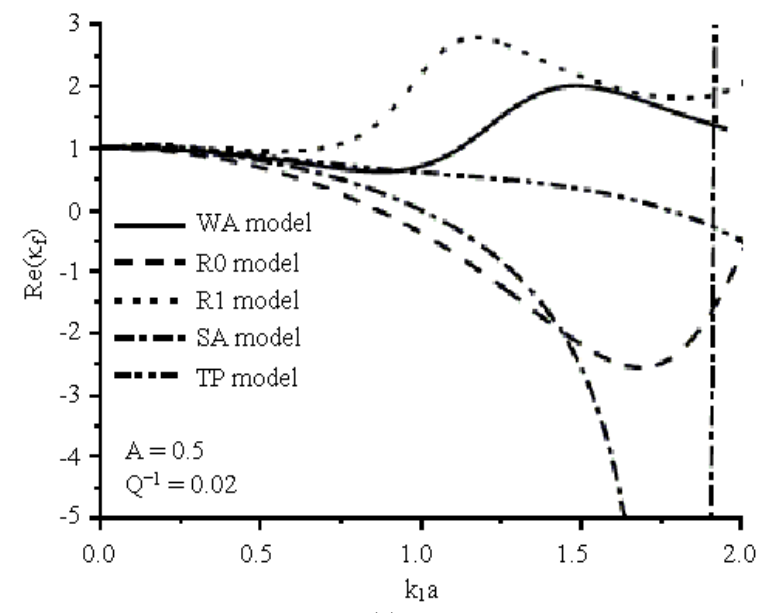

(a)

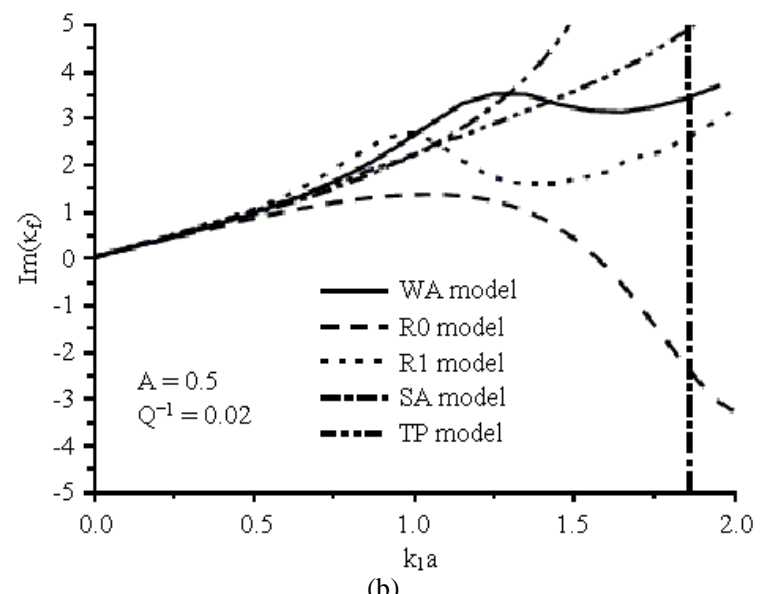

Fig. 3: Influence of contact-displacement conditions on the dynamic contact stiffness at $\mathrm{A}=0.5$ and $v=0.25$ (a) the real part (b) the imaginary part
WA and R1 models predict the reasonable results. WA model includes the influence of the contact displacements throughout the contact interface being related with the contact-pressure distribution. Bycrot, (1956) has verified that the WA model can predict the more accurate results than the R1 model. Therefore, the results for the WA model will be shown in the following analysis.

Anisotropic factor A of single cubic crystals is usually between 0.26 (rubidium iodide) and 8.52 (lithium) (Simmons and Wang, 1971). Figure 4 shows the dependence of DCSF on $\mathrm{k}_{1} \mathrm{a}$ and $\mathrm{A}$ at $v=$ 0.25 . The real and imaginary parts of DCSF are shown in Fig. 4a and b, respectively. The real part of DCSF decreases monotonously with the increase of $\mathrm{k}_{1} \mathrm{a}$ and the imaginary part of DCSF increases as $\mathrm{k}_{1} \mathrm{a}$ increases, which means that the vibration of the sphere will soften the contact stiffness. The real part of dynamic contact stiffness has the limit of anisotropic static contact stiffness $\mathrm{k}_{\mathrm{sa}}$ when $\mathrm{k}_{1} \mathrm{a} \rightarrow 0$.

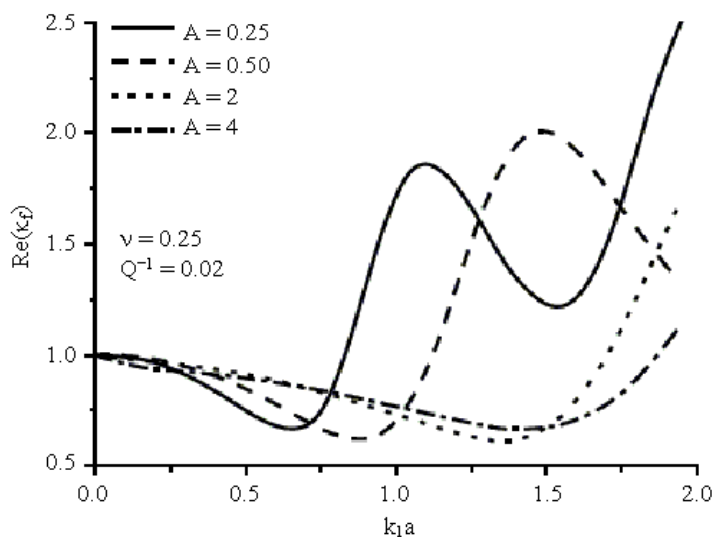

(a)

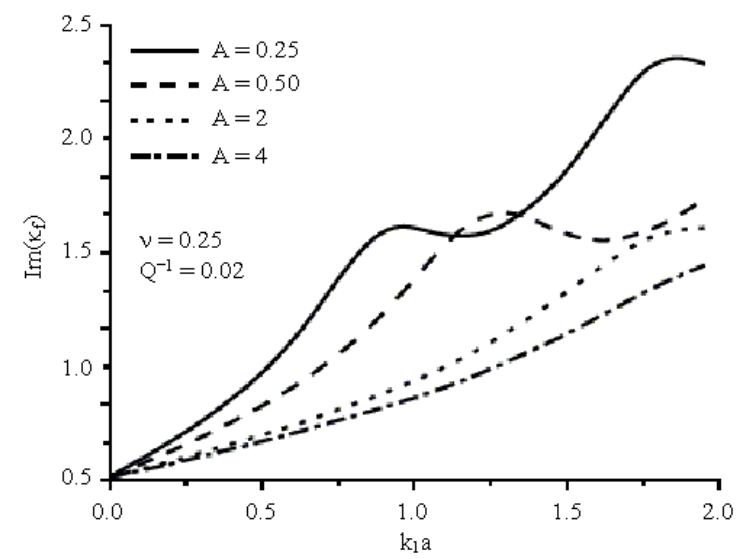

(b)

Fig. 4: Dependence of DCSF on anisotropic factor A and the normalized wave number $\mathrm{k}_{1}$ a at $\mathrm{v}=0.25$ (a) the real part (b) the imaginary part 


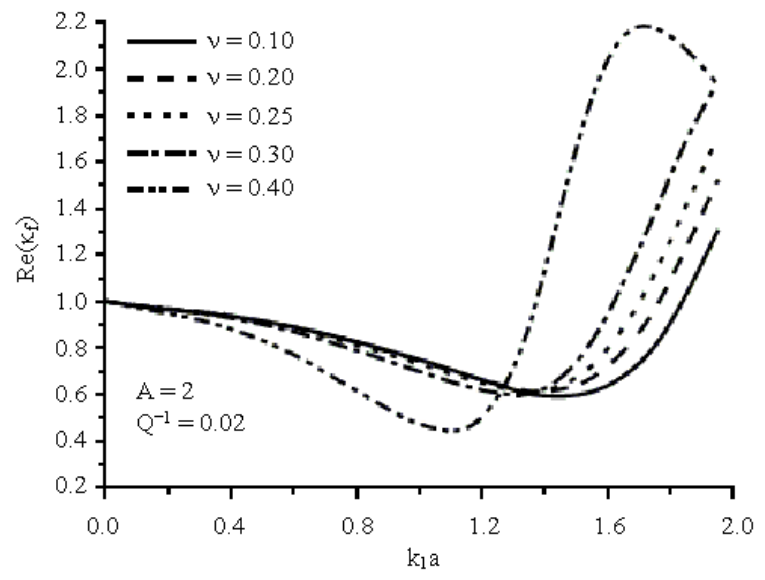

(a)

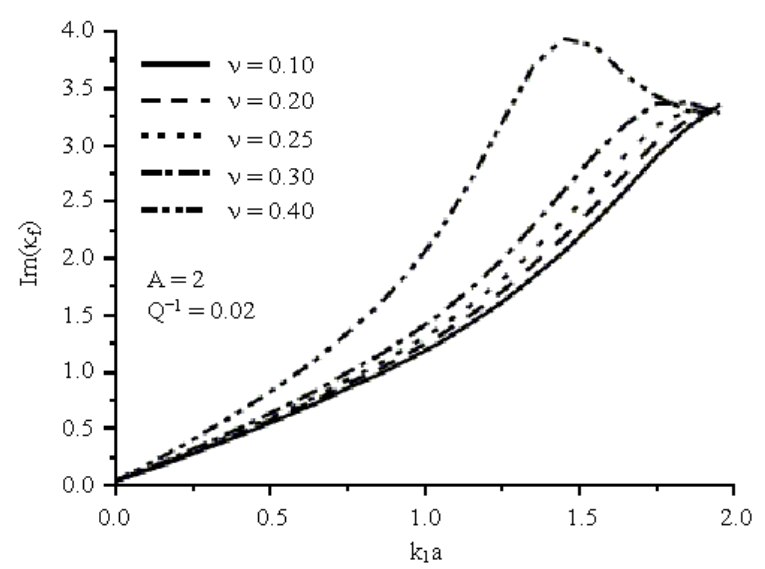

(b)

Fig. 5: Influence of Poisson ratio $v$ and the normalized wave number $\mathrm{k}_{1}$ a on DCSF at $\mathrm{A}=2$ (a) the real part (b) the imaginary part

In RUS, the exciting frequency of the oscillator is between 0.25 and $1.5 \mathrm{MHz}$ and the contact radius is smaller than $30 \mu \mathrm{m}$, which is determined by the biasing force, sphere radius and elastic constants of the solid. For example, when the oscillating rigid sphere contacts the soft cubic grains (rubidium bromide and lead), the normalized wave number $\mathrm{k}_{1} \mathrm{a}$ could reach 0.2 and dynamic contact stiffness is more than $95 \%$ of static stiffness, where we must consider the dynamic characteristic of contact stiffness for quantitative evaluation of elastic modulus in RUS. But for harder cubic grains (diamond, tungsten), $\mathrm{k}_{1} \mathrm{a}$ is much smaller so that we can use static contact stiffness to model dynamic contact.

The DCSFs for $\mathrm{A}=0.25,0.5,2$ and 4 will have the minimum of $0.652,0.617,0.60$ and 0.665 at $\mathrm{k}_{1} \mathrm{a}=0.65$, $0.85,1.35$ and 1.45 , respectively. The DCSFs for
$\mathrm{A}=0.25,0.5$ reach the local maximum of 1.85 and 2.03 at $\mathrm{k}_{1} \mathrm{a}=1.15$ and 1.45 . When $\mathrm{k}_{1} \mathrm{a}<0.6$, with the increase of A, the real part of DCSF increases, but its imaginary part decreases. The influence of anisotropic factor A on the real part of DCSF becomes larger as $k_{1}$ a increases, especially for lower anisotropic factor. When the rigid sphere contacts different orientation surfaces of cubic grain, which show different anisotropic factor. If resonance frequency shifts of the oscillator are used to determine orientation of cubic grains in RUS, the larger operating frequency is better. When $\mathrm{k}_{1} \mathrm{a}>0.6$, the influence of anisotropic factor $\mathrm{A}$ on the dynamic contact stiffness become complex.

Figure 5 shows the influence of $v$ and $\mathrm{k}_{1}$ a on DCSF at $\mathrm{A}=2$. When $\mathrm{k}_{1} \mathrm{a}<1.25$, the real part of DCSF decreases with the increase of $v$; when $\mathrm{k}_{1} \mathrm{a}>1.25$, the real part of DCSF increases with the increase of $v$. The imaginary part of DCSF increases as $v$ increases.

\section{CONCLUSION}

In order to investigate the anisotropy influence of cubic solid on the dynamic Hertzian contact, we present the dynamic contact stiffness of the rigid sphere and the semi-infinite cubic solid by considering the dynamic contact deformation at the contact interface, whose contact radius varies with the oscillation. Assuming that the oscillating force is much less than the biasing force, dynamic contact between the rigid sphere and the cubic solid can be simplified into a dynamic contact of the constant contact radius. The introduction of the different contact-displacement models yields a dynamic contact stiffness. The discussions show that the weighted average model for contact-displacement conditions can depict the dynamic Hertzian contact effectively. It is also shown that dynamic contact stiffness factor depends on normalized wave number $\mathrm{k}_{1} \mathrm{a}$ and anisotropic factor and Poisson's ratio of the cubic solid. The vibration stiffness decreases and the vibration damping increases with the increase of $\mathrm{k}_{1} \mathrm{a}$. The real part of DCSF increases with the increase of an isotropic factor and the decrease of Poisson ratio, but in reverse for the imaginary part, which will benefit the quantitative evaluation of the local surface elastic properties of solids in resonance-type microscopies.

\section{ACKNOWLEDGEMENT}

A part of this study was supported by the National Natural Science Foundation of China (No. 10602053 and No. 50808170), research grants from Institute of Crustal Dynamics (No. ZDJ2007-2) and for overseareturned scholar, Personnel Ministry of China. 
Am. J. Engg. \& Applied Sci., 3 (1): 56-63, 2010

\section{REFERENCES}

Awojobi, A.O. and P. Grootenhuis, 1965. Vibration of rigid bodies on semi elastic media. Philosoph. Trans. R. Soc. Lond. A., 287: 27-63. http://adsabs.harvard.edu/abs/1965RSPSA.287...27 A

Barkan, D.D., 1962. Dynamics of Bases and Foundations. McGraw-Hill Book Co., New York, ISBN: 10: 0070036500, pp: 496.

Bycrot, G.N., 1956. Forced vibration of a rigid circular plate on a semi-infinite elastic space and on an elastic stratum. Philosoph. Trans. R. Soc. Lond., A248: 327-368.

http://rsta.royalsocietypublishing.org/content/248/9 48/327.abstract

Cornejo C'ordova, C.J., 2002. Elastodynamics with hysteretic damping. Ph.D. dissertation, Delft University of Technology, Netherlands.

Frost, H.M., 1974. Action of ultrasound on a viscoelastic solid. Ph.D. dissertation, The University of Vermont, USA. http://adsabs.harvard.edu/abs/1974PhDT........12F

Johnson, K.L., 1985. Contact Mechanics. Cambridge University Press, Cambridge.

Kester, E., U. Rabe, L. Presmanes, Ph. Taihades and W. Arnold, 1999. Measurement of mechanical properties of nanoscaled ferrites using atomic force microscopy at ultrasonic frequencies. Nanostruct. Mater., 12: 779-782. DOI: $10.1016 /$ S09659773(99)00235-4.

Luco, J.E. and R.A. Westmann, 1971. Dynamic response of circular footings. J. Eng. Mech. Divis., 97: 1381-1395. http://cedb.asce.org/cgi/WWWdisplay.cgi?7100078

Nayfeh, A., 1995. Wave Propagation in Layered Anisotropic Media with Application to Composites. 1st Edn., North-Holland, New York, USA., ISBN: 10: 0444890181, pp: 332.

Ogi, H., J. Tian, T. Tada and M. Hirao, 2003. Elasticstiffness mapping by resonance- ultrasound microscopy with isolated piezoelectric oscillator. Applied Phys. Lett., 83: 464-466. DOI: 10.1063/1.1593819

Rabe, U., J. Turner and W. Arnold, 1998. Analysis of the high-frequency response of atomic force microscopy cantilevers. Applied Phys. A., 66: S277-S282.

Simmons, G. and H. Wang, 1971. Single Crystal Elastic Constants and Calculated Aggregate Properties: A Handbook. 2nd Edn., MIT Press, Cambridge, MA., ISBN: 10: 0262190923, pp: 320.
Sung, T.Y., 1953. Vibration in semi-infinite solids due to periodic surface loading. ASTM Symp. Dyn. Test. Soils, 156: 35-63.

Tian, J., H. Ogi, T. Tada, M. Hirao and H. Ledbetter, 2004. Local surface elastic constants by resonantultrasound microscopy. J. Applied Phys., 96: 133-137. DOI: $10.1063 / 1.1755432$

Tian, J., H. Ogi, T. Tada and M. Hirao, 2004. Vibration analysis on Electromagnetic-ResonanceUltrasound Microscopy (ERUM) for determining localized elastic constants of solids. J. Acoustic. Soc. Am., 115: 630-636. DOI: 10.1121/1.1642618

Tian, J., H. Ogi and M. Hirao, 2004. Vibration analysis of an elastic-sphere oscillator contacting semiinfinite viscoelastic solids in resonant ultrasound microscopy. J. Applied Phys., 95: 8366-8375. DOI: 10.1063/1.1737472

Tian, J., H. Ogi and M. Hirao, 2004. Dynamic-contact stiffness at the interface between a vibrating rigid sphere and a semi-infinite visco-elastic solid. IEEE Trans. Ultras. Ferroelect. Freq. Control, 51: 1557-1563. DOI: 10.1109/TUFFC.2004.1367497

Tian, J., H. Ogi, M. Hirao, 2005. Effect of elastic anisotropy on contact stiffness in resonance ultrasound microscopy. Applied Phys. Lett., 87: 2041071-2041073. DOI: 10.1063/1.2131201

Tian, J., H. Ogi, T. Tada and M. Hirao, 2008. Freestanding oscillator for resonance- ultrasound microscopy. IEEE Trans. Ultras. Ferroelect. Freq. Control, 55: 499-502. DOI: 10.1109/TUFFC.2008. 668

Veletsos, A.S. and B. Verbic, 1974. Basic response functions for elastic foundations. J. Eng. Mech. Divis., $\quad 100$ : 189-202. http://cedb.asce.org/cgi/WWWdisplay.cgi?7400254

Yamanaka, K. and S. Nakano, 1998. Quantitative elasticity evaluation by contact resonance in an atomic force microscope. Applied Phys. A., 66: S313-S317.

Yamanaka, K., A. Noguchi, T. Tsuji, T. Koike and T. Goto, 1999. Quantitative material characterization by ultrasonic AFM. Surface Interface Anal., 27: 600-606. DOI: $\quad 10.1002 /($ SICI $1096-9918$ (199905/06)27:5/6<600::AID-SIA508> 3.0.CO;2-W 